\title{
Calculation of smoking rates by dong/eup/myeon unit using small-area estimation in the Community Health Survey
}

\author{
Kay O Lee ${ }^{1}$, Jong Seok Byun ${ }^{2}$, Yang Wha Kang ${ }^{3}$, Yun Sil Ko ${ }^{3}$, Hyo Jin Kim ${ }^{3}$ \\ ${ }^{1}$ Gallup Korea, Seoul; ${ }^{2}$ Department of Applied Statistics, Hanshin University, Osan; ${ }^{3}$ Division of Chronic Disease Control, Korea Centers for \\ Disease Control and Prevention, Cheongju, Korea
}

\section{INTRODUCTION}

The Korean Community Health Survey (CHS), a communitybased nationwide annual survey with the objective of providing important health indicators, is conducted through stratified cluster sampling and computer-assisted personal interviewing. Using the dong/eup/myeon administrative units (hereafter units) and residential structures (apartment or single house) as stratification variables, 900 adults (age $\geq 19$ years) per community health center district (hereafter district) are sampled and proportionally distributed across the units and according to residential structures, followed by selecting tong/ban/ri-level sample points via probability proportionate sampling based on the number of households. From each selected sample point, five households on average are selected by systematic sampling, and individual interviews are conducted with all adults in each household [1]. Although district-level health indicators are produced with a specific level of precision, there is an increasing demand for producing unit-level health indicators. However, the unit sample size varies considerably ranging between tens and several hundreds, and health indicators such as smoking rate for units with fewer than 30 samples produced using conventional statistical estimation methods cannot be used, owing to an exceedingly large sample variance of the estimates. This problem can be addressed by calculating unit-level statistics using a special estimation method such as small-area estimation [2].This paper presents an optimized estimation method using Statistical Analysis System (SAS) codes.

Correspondence: Kay O Lee

Gallup Korea, 70 Sajik-ro, Jongno-gu, Seoul 110-054, Korea

Tel: +82-2-3702-2582, Fax: +82-2-3702-2628, Email: kolee575@hanmail.net

Received: Jan 22, 2015, Accepted: Mar 2, 2015, Published: Mar 2, 2015

This article is available from: http://e-epih.org/

(C) 2015 , Korean Society of Epidemiology

(C) This is an open-access article distributed under the terms of the Creative Commons Attribution License (http://creativecommons.org/licenses/by/3.0/), which permits unrestricted use, distribution, and reproduction in any medium, provided the original work is properly cited.

\section{Small-area estimation}

Small-area estimation is an estimation method designed to produce statistics for small survey areas not included in the sample design for statistics and having unusable high-variance estimates owing to excessively small sample sizes, through supplementary use of surrounding area survey information, auxiliary information from other sources, or statistical model of the population $[3,4]$.

Given that the sample design for the Community Health Service is intended to produce district-level health indicators, smallarea estimation is needed for producing reliable unit-level health indicators. The following describes the small-area estimation methods for producing unit-level health indicators [5].

\section{Direct estimator}

A direct estimator uses only data obtained from the units concerned to produce unit-level health indicators. Each observation included in the survey data sets is given a weight item by item; the sample design-based direct estimator and its variance can be expressed by the following estimation equation, using weighted and observed values:

$$
\hat{\bar{Y}}_{\mathrm{D}}^{\mathrm{i}}=\frac{\sum_{j=1}^{n_{i}} w_{j}^{i} y_{j}^{i}}{\sum_{j=1}^{n_{i}} w_{j}^{i}}
$$

where $n_{i}$ is the sample size of unit $i, w_{j}^{i}$ is the multiplier reflecting sample and response rates, and $y_{j}^{i}$ is the observed value.

The variance of the estimator shown in Equation (1) can be obtained using Equation (2), as follows:

$$
\widehat{\operatorname{Var}}\left(\hat{\bar{Y}}_{D}^{i}\right)=\frac{1}{n_{i}\left(n_{i}-1\right) \bar{w}_{i}^{2}} \sum_{j=1}^{n_{i}}\left(w_{j}^{i}\right)^{2}\left(y_{j}^{i}-\widehat{R}_{\imath}\right)^{2}
$$

where $\widehat{R}_{\iota}=\frac{\sum_{j=1}^{n_{i}} w_{j}^{i} y_{j}^{i}}{\sum_{j=1}^{n_{i}} w_{j}^{i}}$ and $\bar{w}_{\mathrm{i}}=\frac{1}{n_{i}} \sum_{j=1}^{n_{i}} w_{j}^{i}$

\section{Synthetic estimator}

A synthetic estimator can yield more accurate unit-level estimates by using various auxiliary data, such as sex, age, and reg- 
istered population, for each unit within a district [4,5]. After grouping the units into 2 to 3 homogeneous clusters, using their social environments and population ratios as clustering variables, under the assumption that the units of the same cluster might have similar sex-dependent/age-dependent health indicators, such as smoking rate, unit-level smoking rates can be calculated as follows, by combining smoking rates by sex and age group and by the number of people registered:

$$
\hat{\bar{Y}}_{\mathrm{S}}^{\mathrm{i}}=\frac{\sum_{k=1}^{n_{c}} r_{j k} N_{j k}^{i}}{\sum_{j=1}^{n_{C}} N_{j k}^{i}}
$$

where $\mathrm{r}_{\mathrm{jk}}=\frac{\sum_{l 1}^{n_{g}} w_{j k l} y_{j k l}}{\sum_{l=1}^{n_{g}} w_{j k l}}$ is the average estimate of category $k$ within cluster $j, N_{j k}^{i}$ is unit $i$ within cluster $j$, registered population of category $k, n_{g}$ is the number of samples in category $k$ within cluster $j$, and $n_{c}$ is the number of categories within cluster $j$.

The variance of the estimate shown in Equation (3) can be obtained using Equation (4):

$$
\begin{gathered}
\widehat{\operatorname{Var}}\left(\hat{\bar{Y}}_{S}^{i}\right)=\sum_{k=1}^{n_{c}}\left(Z_{j k}^{i}\right)^{2} \widehat{\operatorname{Var}}\left(r_{j k}^{i}\right) \\
=\sum_{k=1}^{n_{c}} \frac{\left(Z_{j k}^{i}\right)^{2}}{n_{g}\left(n_{g}-1\right) \bar{w}_{j k}^{2}} \sum_{l=1}^{n_{g}}\left(w_{j k l}\right)^{2}\left(y_{j k l}-r_{j k}\right)^{2}
\end{gathered}
$$

where $z_{j k}^{i}=\frac{N_{j k}^{i}}{\sum_{k=1}^{n_{c}} N_{j k}^{i}}$

While a synthetic estimator is potentially biased, the bias is considered negligible, given that the clustering of units within the same district ensures the sex/age category characteristics across units. If the bias exceeds a negligible level, attention must be paid to the clustering, because the equation for estimating the variance shown in Equation (4) tends to underestimate the estimation error of the estimator of Equation (3).

\section{Composite estimator}

Although the direct estimator shown in Equation (1) is unbiased, its estimates are not reliable, with unacceptably large standard error resulting from the small sample size, whereas the synthetic estimator shown in Equation (3) is biased.These problems can be addressed using a hybrid approach of obtaining more reliable estimates from the weighted average of the two estimators. The formula of weighted estimate average shown in Equations (1) and (3) is referred to as a composite estimator and can be calculated as follows:

$$
\hat{\bar{Y}}_{C}^{i}=\alpha \hat{\bar{Y}}_{D}^{i}+(1-\alpha) \widehat{\widehat{Y_{S}^{l}}}
$$

where $\alpha$ is the weight that minimizes $\operatorname{MSE}\left(\widehat{\widehat{Y_{C}^{l}}}\right)$ and can be calculated as follows:

$$
\alpha=\frac{\widehat{\operatorname{Var}}\left(\hat{\bar{Y}}_{S}^{i}\right)}{\widehat{\operatorname{Var}}\left(\widehat{Y}_{D}^{i}\right)+\widehat{\operatorname{Var}}\left(\hat{Y}_{S}^{i}\right)}
$$

While the optimal value of $\alpha$ is expected to be the one that minimizes the root mean square error of $\hat{Y}_{C}^{i}, \alpha$ is calculated using Equation (6) under the assumption that clustered units are sufficiently homogeneous to ignore the bias of the synthetic estimator $\hat{\bar{Y}}_{S}^{i}$, and that the direct and synthetic estimators are independent of each other.

\section{Calculation of smoking rate using statistical analysis system}

The following describes the calculation process of the unit-level smoking rates using the 2013 CHS data by applying small-area estimation to the calculation of smoking rates for the 22 dongs in Gangnam-gu, Seoul.

\section{Direct estimator}

The sample size distribution of the 22 dongs of Gangnam-gu included in the 2013 CHS shows that the dongs with the smallest and largest sample sizes are Gaepo 4- dong $(n=24)$ and Yeoksam 1-dong $(n=58)$. The smoking rates by dong were calculated using the direct estimators shown in Equation (1) and the variance estimation Equation (2) using SAS code as follows. The R-code program was presented in a previous study [6].

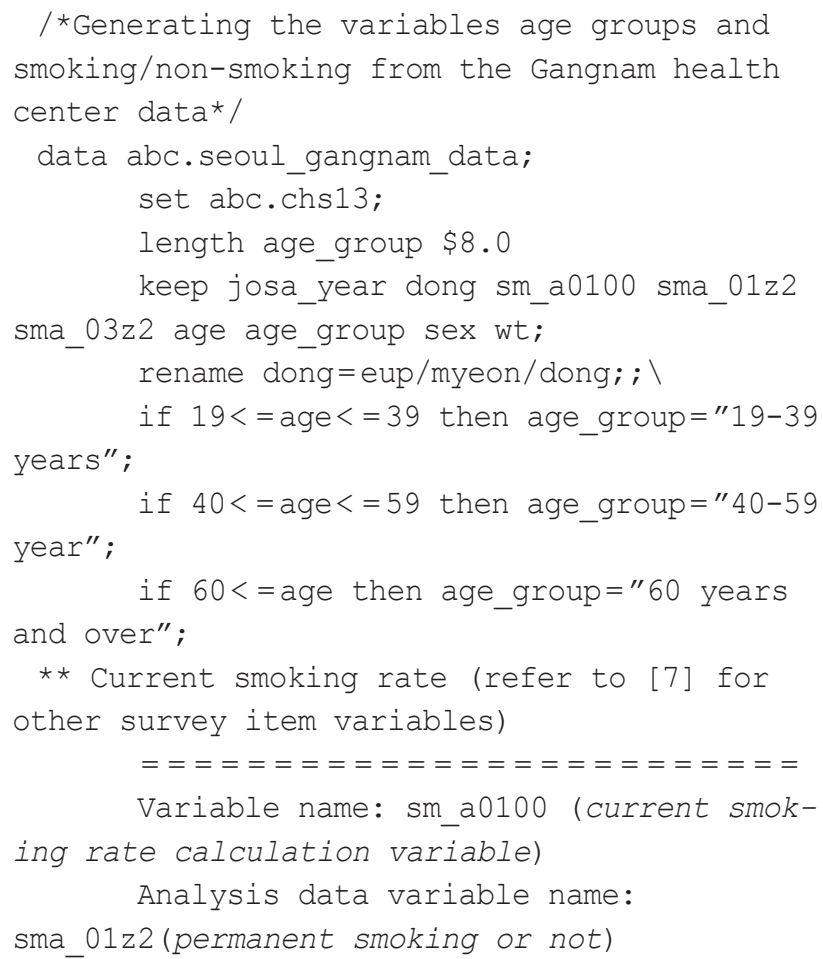




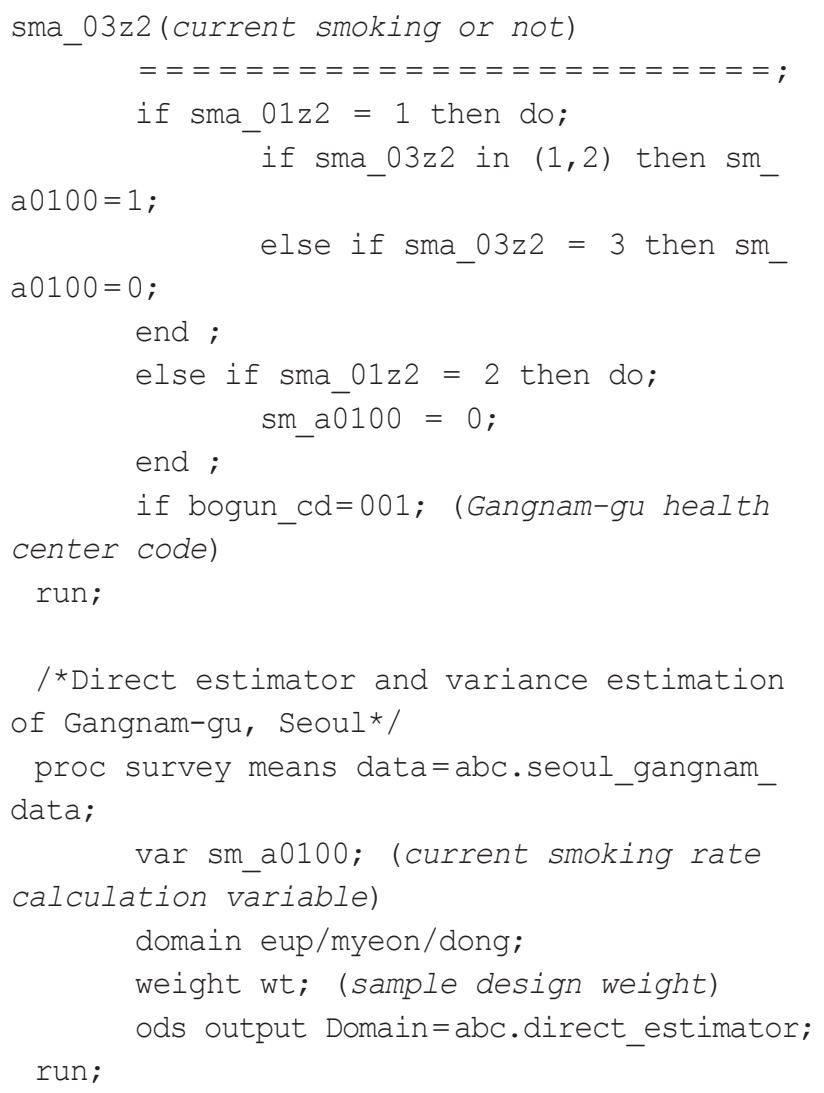

\section{Synthetic estimator}

To calculate the synthetic estimators of smoking rates by dong, the 22 dongs of Gangnam-gu were grouped into three clusters. After calculating the smoking rates of each cluster by sex and age group (19 to 39,40 to $59, \geq 60$ ), the smoking rates and variances by dong were calculated using Equations (3) and (4), respectively, as follows:

(1) Calculation of the registered population ratios by sex and age group of 22 dongs as of the end of July 2013

(2) Grouping 22 dongs in three clusters using the k-means method, with the population ratio and smoking rate as clustering variables, and with a homogeneity check of the clustered groups against the registered population size and socioeconomic environment

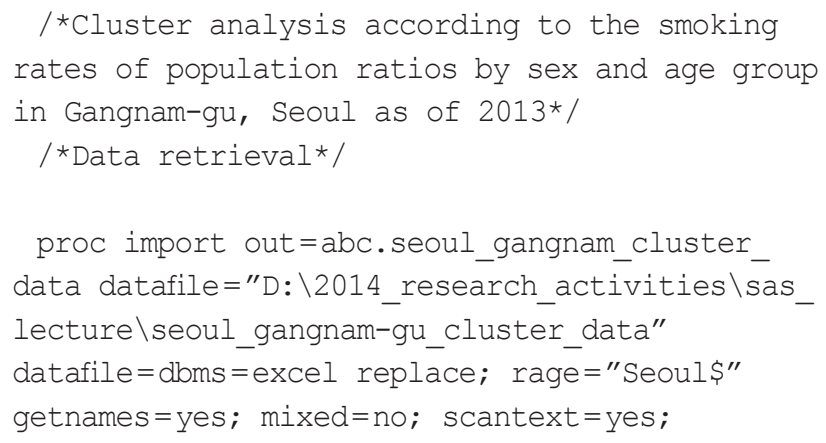

Table 1. Registered population ratios by sex and age group for each dong

\begin{tabular}{|c|c|c|c|c|c|c|}
\hline \multirow{2}{*}{ Dong } & \multicolumn{3}{|c|}{ Male } & \multicolumn{3}{|c|}{ Female } \\
\hline & $19-39$ & $40-59$ & $\geq 60$ & $19-39$ & $40-59$ & $\geq 60$ \\
\hline Shinsa-dong & 0.188 & 0.174 & 0.094 & 0.243 & 0.192 & 0.109 \\
\hline Nonhyeon 1-dong & 0.241 & 0.149 & 0.066 & 0.309 & 0.155 & 0.080 \\
\hline Nonhyeon 2-dong & 0.220 & 0.158 & 0.083 & 0.268 & 0.175 & 0.095 \\
\hline Apgujeong-dong & 0.172 & 0.178 & 0.106 & 0.209 & 0.206 & 0.128 \\
\hline Cheongdam-dong & 0.198 & 0.182 & 0.085 & 0.228 & 0.209 & 0.098 \\
\hline Samsung 1-dong & 0.203 & 0.191 & 0.091 & 0.200 & 0.217 & 0.099 \\
\hline Samsung 2-dong & 0.203 & 0.189 & 0.069 & 0.257 & 0.198 & 0.084 \\
\hline Daechi 1-dong & 0.140 & 0.262 & 0.073 & 0.159 & 0.287 & 0.078 \\
\hline Daechi 2-dong & 0.175 & 0.238 & 0.074 & 0.173 & 0.259 & 0.080 \\
\hline Daechi 4-dong & 0.211 & 0.188 & 0.058 & 0.257 & 0.216 & 0.069 \\
\hline Yeoksam 1-dong & 0.256 & 0.155 & 0.064 & 0.310 & 0.145 & 0.070 \\
\hline Yeoksam 2-dong & 0.194 & 0.199 & 0.067 & 0.247 & 0.210 & 0.083 \\
\hline Dogok 1-dong & 0.198 & 0.195 & 0.081 & 0.223 & 0.214 & 0.090 \\
\hline Dogok 2-dong & 0.167 & 0.211 & 0.090 & 0.203 & 0.239 & 0.091 \\
\hline Gaepo 1-dong & 0.193 & 0.193 & 0.087 & 0.189 & 0.234 & 0.105 \\
\hline Gaepo 2-dong & 0.209 & 0.204 & 0.061 & 0.211 & 0.233 & 0.083 \\
\hline Gaepo 4-dong & 0.207 & 0.204 & 0.070 & 0.208 & 0.226 & 0.085 \\
\hline Segok-dong & 0.188 & 0.193 & 0.105 & 0.188 & 0.194 & 0.132 \\
\hline Ilwonbon-dong & 0.182 & 0.226 & 0.065 & 0.195 & 0.253 & 0.079 \\
\hline Ilwon 1-dong & 0.209 & 0.179 & 0.091 & 0.196 & 0.205 & 0.120 \\
\hline Ilwon 2-dong & 0.205 & 0.189 & 0.077 & 0.193 & 0.221 & 0.114 \\
\hline Sooseo-dong & 0.205 & 0.144 & 0.101 & 0.185 & 0.191 & 0.173 \\
\hline
\end{tabular}




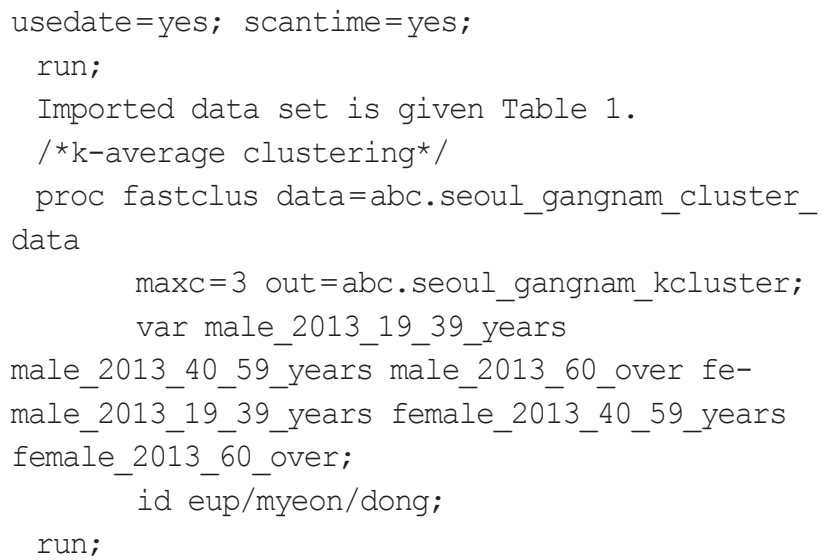

(3) Smoking rates of each cluster by sex and age group /*Corrected cluster integration in data from Gangnam-gu, Seoul*/

proc sort data=abc.seoul_gangnam_data; by eup/ myeon/dong; run;

proc sort data=abc.seoul_gangnam_rcluster; by eup/myeon/dong; run;

data abc.seoul_gangnam_data2; merge abc.seoul_gangnam_data abc.seoul_ gangnam_rcluster; by eup/myeon/dong; group $1=$ corrected

cluster||"_"| sex||"_"| |age_group; group1=compress (group1) ;

run;

proc print data $=a b c$. seoul gangnam data2; run; The corrected data set is displayed in Table 2. $/{ }^{*}$ Calculation of the estimated smoking rates of each cluster by sex and age group*/

proc surveymeans data $=a b c$. seoul_gangnam_data2 mean;

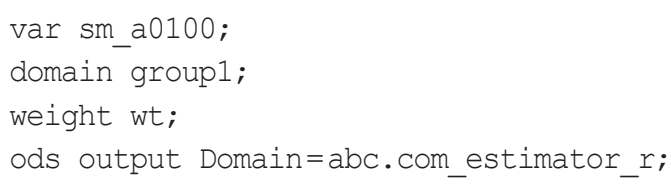

The results of calculation are contained in Table 3 .

(4) Calculation of synthetic estimates and variances of the smoking rates in 22 dongs

/*Preparation of the data for the clusterlevel sex/age-dependent smoking rate estimation*/

/*Corrected cluster integration in data from Gangnam-gu, Seoul*/

proc sort data=abc.seoul_gangnam_data; by eup/myeon/dong; run;

proc sort data=abc.seoul_gangnam_rcluster; by eup/myeon/dong; run;

data abc.seoul_gangnam_data2;

merge abc.seoul_gangnam_data abc.seoul_ gangnam_rcluster;

Table 2. Examples of data structure, including corrected cluster, sex and age group, and variables for calculation smoking rate

\begin{tabular}{|c|c|c|c|c|c|c|c|c|c|c|c|}
\hline Observation & Eup/myeon/dong & Sex & $\begin{array}{l}\text { sma } \\
01 z 2\end{array}$ & $\begin{array}{l}\text { sma } \\
03 z 2\end{array}$ & Age & wt & $\begin{array}{l}\text { Age_} \\
\text { group }\end{array}$ & $\begin{array}{l}\text { sma } \\
0100\end{array}$ & Cluster & $\begin{array}{c}\text { Corrected } \\
\text { cluster }\end{array}$ & $\begin{array}{c}\text { Group1 } \\
\text { (age) }\end{array}$ \\
\hline 1 & Nonhyeon 1-dong & $M$ & 1 & 1 & 26 & 592.15 & $19-39$ & 1 & 2 & 1 & 1_1_19-39 \\
\hline 2 & Nonhyeon 1-dong & M & 1 & 1 & 36 & 647.70 & $19-39$ & 1 & 2 & 1 & 1_1_19-39 \\
\hline 3 & Nonhyeon 1-dong & M & 1 & 1 & 35 & 647.46 & $19-39$ & 1 & 2 & 1 & 1_1_19-39 \\
\hline 4 & Nonhyeon 1-dong & M & 1 & 1 & 25 & 592.15 & 19-39 & 1 & 2 & 1 & 1_1_19-39 \\
\hline 5 & Nonhyeon 1-dong & M & 2 & . & 19 & 592.15 & $19-39$ & 0 & 2 & 1 & 1_1_19-39 \\
\hline 6 & Nonhyeon 1-dong & M & 2 & 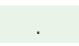 & 32 & 647.46 & $19-39$ & 0 & 2 & 1 & 1_1_19-39 \\
\hline 7 & Nonhyeon 1-dong & M & 1 & 1 & 32 & 647.46 & $19-39$ & 1 & 2 & 1 & 1_1_19-39 \\
\hline 8 & Nonhyeon 1-dong & M & 1 & 1 & 39 & 647.46 & $19-39$ & 1 & 2 & 1 & 1_1_19-39 \\
\hline
\end{tabular}

sma, smoking variable; wt, weight; $M$, male. 
Table 3. Current smoking rate estimates and standard errors by group (cluster, sex/age)

\begin{tabular}{llll}
\hline Group (age) & Variable & Mean & Standard error \\
\hline 1_1_19-39 & sm_a0100 & 0.465 & 0.074 \\
1_1_40-59 & sm_a0100 & 0.474 & 0.076 \\
1_1_ 260 & sm_a0100 & 0.242 & 0.085 \\
1_2_19-39 & sm_a0100 & 0.168 & 0.056 \\
1_2_40-59 & sm_a0100 & 0.075 & 0.032 \\
1_2_ 260 & sm_a0100 & 0.032 & 0.031 \\
2_1_19-39 & sm_a0100 & 0.364 & 0.074 \\
2_1_40-59 & sm_a0100 & 0.419 & 0.062 \\
2_1_ 260 & sm_a0100 & 0.160 & 0.062 \\
2_2_19-39 & sm_a0100 & 0.137 & 0.048 \\
2_2_40-59 & sm_a0100 & 0.057 & 0.030 \\
2_2_ 260 & sm_a0100 & 0.0 & 0.0 \\
3_1_19-39 & sm_a0100 & 0.413 & 0.068 \\
3_1_40-59 & sm_a0100 & 0.317 & 0.065 \\
3_1_ 260 & sm_a0100 & 0.207 & 0.085 \\
3_2_19-39 & sm_a0100 & 0.065 & 0.038 \\
3_2_40-59 & sm_a0100 & 0.0 & 0.0 \\
3_2_ 260 & sm_a0100 & 0.134 & 0.056 \\
\hline
\end{tabular}

by eup/myeon/dong;

group $1=$ corrected

cluster||"_"||sex||"_"| |age_group;

group1=compress (group1) ;

run;

/*Synthetic estimator for the 22 dongs in Gangnam-gu, Seoul* /

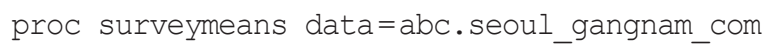
mean;

domain eup/myeon/dong;

var mean;

weighted population;

ods output domain=abc.seoul_gangnam_comestimator_mean;

run;

Synthetic estimates are displayed in Table 4.

/*Variance estimation of the synthetic estimators for each dong*/

proc sort data=abc.seoul_gangnam_data2; by group1; run;

data abc.seoul_gangnam_comvar;

merge abc.seoul_gangnam data2 abc.com estimator_r;

by group1;

sum wj yj $r j=\left(\left(w t^{\star} w t\right) *((s m a 0100-\right.$

mean $) *($ sm a0100-mean $)))$;

run;

/*Total weights of the k-category within a
Table 4. Current smoking rate estimates and standard errors

\begin{tabular}{lcc}
\hline Dong & Mean & Standard error \\
\hline Gaepo 1-dong & 0.185 & 0.071 \\
Gaepo 2-dong & 0.188 & 0.074 \\
Gaepo 4-dong & 0.190 & 0.073 \\
Nonhyeon 1-dong & 0.265 & 0.074 \\
Nonhyeon 2-dong & 0.259 & 0.073 \\
Daechi 1-dong & 0.211 & 0.076 \\
Daechi 2-dong & 0.214 & 0.072 \\
Daechi 4-dong & 0.213 & 0.066 \\
Dogok 1-dong & 0.210 & 0.066 \\
Dogok 2-dong & 0.205 & 0.068 \\
Samsung 1-dong & 0.260 & 0.077 \\
Samsung 2-dong & 0.261 & 0.075 \\
Segok-dong & 0.190 & 0.066 \\
Sooseo-dong & 0.187 & 0.066 \\
Shinsa-dong & 0.251 & 0.073 \\
Apgujeong-dong & 0.245 & 0.073 \\
Yeoksam 1-dong & 0.219 & 0.062 \\
Yeoksam 2-dong & 0.211 & 0.066 \\
Ilwon 1-dong & 0.190 & 0.070 \\
Ilwon 2-dong & 0.189 & 0.071 \\
Ilwonbon-dong & 0.184 & 0.073 \\
Cheongdam-dong & 0.256 & 0.075 \\
\hline
\end{tabular}

cluster* /

proc surveymeans data $=a b c$.seoul_gangnam_comvar;

$$
\text { domain group1; }
$$

var wt;

ods output domain $=a b c$. seoul_gangnam_

comvar_1;

run;

/*Total for the intragroup k-category variance estimation*/

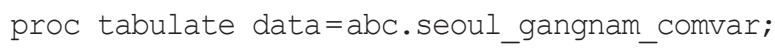
class group1;

var sum_wj_yj_rj;

table group1*sum_wj_yj_rj;

ods output table $=a b c$. seoul_gangnam_com-

var_2;

run;

data abc.seoul_gangnam_comvar_datal;

merge abc.seoul_gangnam_pop abc.seoul_

gangnam_comvar_1 abc.seoul_gangnam_comvar_2;

by group1;

keep eup/myeon/dong cluster sex age-

group population N Mean sum_wj_yj_rj_sum

group1;

run;

Table 5 contains the generated data set. 
proc surveymeans data $=a b c$. seoul_gangnam_comvar_datal sum;

domain eup/myeon/dong;

var population;

ods output domain=abc.seoul_gangnam comvar_data2;

run;

proc sort data=abc.seoul_gangnam_comvar_ data1; by eup/myeon/dong; run;

proc sort data =abc.seoul_gangnam_comvar_ data2; by eup/myeon/dong; run;

/*Synthetic estimator variance estimation*/

data abc.seoul gangnam comvar data;

merge abc.seoul_gangnam_comvar_datal

abc.seoul_gangnam_comvar_data2;

by eup/myeon/dong;

drop varname varlabel stddev DomainLa-

bel;

$$
\begin{aligned}
& Z j k=\text { Population/Sum; } \\
& \operatorname{Var}=\left(\left(Z j k^{*} Z j k\right) /\left(\left(N^{*}(N-\right.\right.\right.
\end{aligned}
$$

$1)) *($ mean*mean $))) *$ sum_wj_yj_rj_sum;

run;

Table 6 contains variance of syntyetic estimate.

proc surveymeans data $=a b c$. seoul_gangnam_comvar_data sum; domain eup/myeon/dong;

var var;

ods output domain $=a b c$. seoul_gangnam

comvariance;

run;

(5) Data set generation by integrating the synthetic estimates and variance estimates for the smoking rates in all 22 dongs

/*Data integration of dong-level synthetic

estimates and variance estimates*/

/*Synthetic estimator and variance*/

data abc.seoul gangnam estimator com;

merge abc.seoul_gangnam_comestimator_mean

abc.seoul gangnam comvariance;

by eup/myeon/dong;

drop DomainLabel VarName stderr StdDev; rename mean $=Y \_s$ sum $=$ var_Y_S;

run;

\section{Composite estimator}

The dong-level direct and synthetic estimates of smoking rates are combined as weighted averages to calculate the composite estimates, thereby applying the following three methods for calculating weighted values.

First, $\alpha_{i}$ for minimizing the mean square error of the composite estimator $\operatorname{MSE}\left(\hat{\bar{Y}}_{c}^{\mathrm{i}}\right)$ hown in Equation (5) is expressed as follows:

\begin{tabular}{|c|c|c|c|c|c|c|c|c|c|}
\hline Observation & Eup/myeon/dong & $\begin{array}{c}\text { Corrected } \\
\text { cluster }\end{array}$ & Sex & Age & Population & $\begin{array}{c}\text { Group1 } \\
\text { (age) }\end{array}$ & $\mathrm{n}$ & $\begin{array}{l}\text { Mean } \\
(w t)\end{array}$ & $\begin{array}{l}\text { sum_wj_yj_rj_ } \\
\text { Sum }\end{array}$ \\
\hline 1 & Shinsa-dong & 1 & M & $19-39$ & 3,071 & 1_1_19-39 & 47 & 629.36 & 4825692 \\
\hline 2 & Nonhyeon 1-dong & 1 & M & $19-39$ & 5,629 & 1_1_19-39 & 47 & 629.36 & 4825692 \\
\hline 3 & Nonhyeon 2-dong & 1 & M & $19-39$ & 4,267 & 1_1_19-39 & 47 & 629.36 & 4825692 \\
\hline 4 & Apgujeong-dong & 1 & M & $19-39$ & 4,014 & 1_1_19-39 & 47 & 629.36 & 4825692 \\
\hline 5 & Cheongdam-dong & 1 & M & $19-39$ & 5,060 & 1_1_19-39 & 47 & 629.36 & 4825692 \\
\hline 6 & Samsung 1-dong & 1 & M & $19-39$ & 2,577 & 1_1_19-39 & 47 & 629.36 & 4825692 \\
\hline 7 & Samsung 2-dong & 1 & M & $19-39$ & 5,224 & 1_1_19-39 & 47 & 629.36 & 4825692 \\
\hline 8 & Shinsa-dong & 1 & M & $40-59$ & 2,841 & 1_1_40-59 & 48 & 470.96 & 2914779 \\
\hline 9 & Nonhyeon 1-dong & 1 & M & $40-59$ & 3,476 & 1_1_40-59 & 48 & 470.96 & 2914779 \\
\hline 10 & Nonhyeon 2-dong & 1 & M & $40-59$ & 3,061 & 1_1_40-59 & 48 & 470.96 & 2914779 \\
\hline 11 & Apgujeong-dong & 1 & M & $40-59$ & 4,153 & 1_1_40-59 & 48 & 470.96 & 2914779 \\
\hline 12 & Cheongdam-dong & 1 & M & $40-59$ & 4,659 & 1_1_40-59 & 48 & 470.96 & 2914779 \\
\hline 13 & Samsung 1-dong & 1 & M & $40-59$ & 2,427 & 1_1_40-59 & 48 & 470.96 & 2914779 \\
\hline 14 & Samsung 2-dong & 1 & M & $40-59$ & 4,852 & 1_1_40-59 & 48 & 470.96 & 2914779 \\
\hline 15 & Shinsa-dong & 1 & M & $\geq 60$ & 1,527 & 1_1_z60 & 27 & 414.07 & 898420.2 \\
\hline 16 & Nonhyeon 1-dong & 1 & M & $\geq 60$ & 1,540 & 1_1_z60 & 27 & 414.07 & 898420.2 \\
\hline 17 & Nonhyeon 2-dong & 1 & M & $\geq 60$ & 1,613 & 1_1_z60 & 27 & 414.07 & 898420.2 \\
\hline 18 & Apgujeong-dong & 1 & M & $\geq 60$ & 2,462 & 1_1_z60 & 27 & 414.07 & 898420.2 \\
\hline 19 & Cheongdam-dong & 1 & M & $\geq 60$ & 2,179 & 1_1_z60 & 27 & 414.07 & 898420.2 \\
\hline
\end{tabular}

Table 5. Variance of synthetic estimates by group (cluster, sex/age group)

wt, weight; wj, weight; yj, observed value; rj, smoking rate; $M$, male. 
Table 6. Variance calculation of synthetic estimates by dong and group

\begin{tabular}{|c|c|c|c|c|c|c|c|c|c|c|c|c|}
\hline $\begin{array}{l}\text { Observa- } \\
\text { tion }\end{array}$ & Eup/myeon/dong & $\begin{array}{c}\text { Corrected } \\
\text { cluster }\end{array}$ & Sex & Age & Population & Group1 & $\mathrm{n}$ & Mean & $\begin{array}{c}\text { sum_wj_yj_rj_ } \\
\text { Sum }\end{array}$ & Sum & Zjk & Var \\
\hline 1 & Gaepo 1-dong & 3 & M & $19-39$ & 3,677 & 3_1_19-39 & 54 & 615.277 & 5180109 & 19069 & 0.193 & 0.000178 \\
\hline 2 & Gaepo 1-dong & 3 & M & $40-59$ & 3,677 & 3_1_40-59 & 58 & 461.391 & 3071663 & 19069 & 0.193 & 0.000162 \\
\hline 3 & Gaepo 1-dong & 3 & M & $\geq 60$ & 1,659 & $3 \_1 \_\geq 60$ & 29 & 406.868 & 1009267 & 19069 & 0.087 & 5.68E-05 \\
\hline 4 & Gaepo 1-dong & 3 & $\mathrm{~F}$ & 19-39 & 3,601 & 3_2_19-39 & 64 & 583.223 & 2031942 & 19069 & 0.189 & 5.28E-05 \\
\hline 5 & Gaepo 1-dong & 3 & $\mathrm{~F}$ & $40-59$ & 4,455 & 3_2_40-59 & 78 & 419.521 & 0 & 19069 & 0.233 & 0.00 \\
\hline 6 & Gaepo 1-dong & 3 & $\mathrm{~F}$ & $\geq 60$ & 2,000 & 3_2_ $\geq 60$ & 37 & 411.813 & 728625.3 & 19069 & 0.105 & 3.55E-05 \\
\hline 7 & Gaepo 2-dong & 3 & M & $19-39$ & 5,720 & 3_1_19-39 & 54 & 615.267 & 5180109 & 27359 & 0.209 & 0.000209 \\
\hline 8 & Gaepo 2-dong & 3 & M & $40-59$ & 5,590 & 3_1_40-59 & 58 & 461.391 & 3071663 & 27359 & 0.204 & 0.000182 \\
\hline 9 & Gaepo 2-dong & 3 & M & $\geq 60$ & 1,656 & 3_1_z60 & 29 & 406.868 & 1009267 & 27359 & 0.060 & 2.75E-05 \\
\hline 10 & Gaepo 2-dong & 3 & $\mathrm{~F}$ & $19-39$ & 5,760 & 3_2_19-39 & 64 & 583.223 & 2031942 & 27359 & 0.210 & 6.57E-05 \\
\hline 11 & Gaepo 2-dong & 3 & $\mathrm{~F}$ & $40-59$ & 6,368 & 3_2_40-59 & 78 & 419.521 & 0 & 27359 & 0.233 & 0.00 \\
\hline 12 & Gaepo 2-dong & 3 & $\mathrm{~F}$ & $\geq 60$ & 2,265 & $3 \_2 \_\geq 60$ & 37 & 411.813 & 728625.3 & 27359 & 0.083 & 2.21E-05 \\
\hline
\end{tabular}

wj, weight; yj, observed value; rj, smoking rate; Zjk, proportion of registered population of category k; Var, variance; M, male; F, female.

$$
\alpha_{\mathrm{i}(\mathrm{opt})}=\frac{\operatorname{MSE}\left(\hat{\bar{Y}}_{S}^{i}\right)}{\operatorname{MSE}\left(\hat{\bar{Y}}_{S}^{i}\right)+V\left(\hat{\bar{Y}}_{D}^{i}\right)}
$$

The estimated value of the optimal weight $\alpha_{i(o p t)}$ is calculated as follows:

$$
\hat{\alpha}_{i(o p t)}=\frac{M S E\left(\hat{\bar{Y}}_{S}^{i}\right)}{\left(\hat{Y}_{S}^{i}-\widehat{Y^{l}}\right)^{2}}
$$

The weight minimizing the mean MSE $\left(\hat{\bar{Y}}_{S}^{i}\right)$ using the initial common weight $\alpha$ as an approach to giving the common weight to all small areas is obtained as follows:

$$
\hat{\alpha}(o p t)=1-\frac{\sum_{i} \widehat{V}\left(\hat{\bar{Y}}_{D}^{i}\right)}{\sum_{i}\left(\hat{\bar{Y}}_{S}^{i}-\hat{\bar{Y}}_{i}\right)^{2}}
$$

The weight dependent on the sample size assigned to each small area is calculated as follows:

$$
\alpha_{\mathrm{i}}(\delta)=\left(\begin{array}{cc}
1, & \widehat{N}_{i} \geqq \delta N_{i} \\
\frac{\widetilde{N}_{i}}{\delta N_{i}} & (\text { an so on })
\end{array}\right)
$$

where $N \_i$ is the size of small area $i, \widehat{N}_{i}=N\left(\frac{n_{i}}{n}\right) . \widetilde{N}_{i}$ is the direct estimator, and $\delta$ is the value adjusting the synthetic estimator's contribution, thus a subjectively determined value. The Canadian Labor Force Survey, for example, uses $\delta=2 / 3$, which is also applied to this calculation [8].

After calculating the composite estimators of the dong-level smoking rates with each of the three types of weight presented above, an optimal estimation method is selected. The composite estimates are calculated in the following procedure.

(1) Data set generation by integrating the direct estimates $\left(\mathrm{Y}_{-} \mathrm{d}\right)$ and synthetic estimates (Y_s) calculated for the 22 dongs

/Integration of direct and synthetic estimators (estimates and variance)*/

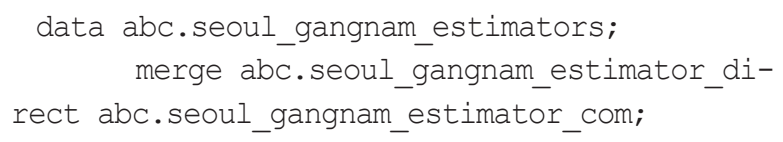

\section{run;}

by eup/myeon/dong;

Table 7 contains the direct and synthetic estimates.

(2) Calculation of composite estimates using the first weight (Y_c1)

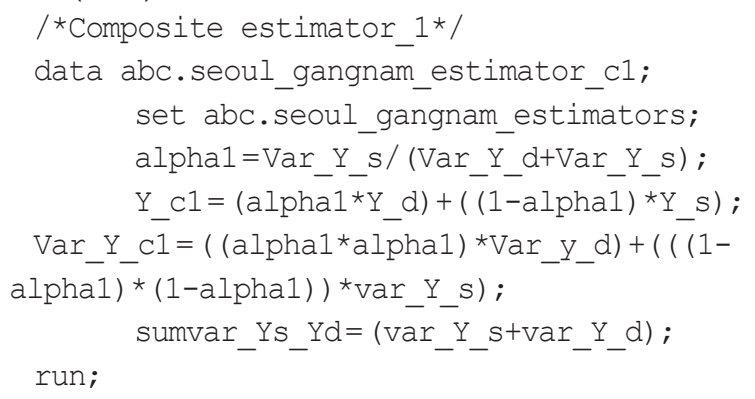

/*Direct estimator variance by corrected cluster and direct estimator variance + composite estimator*/

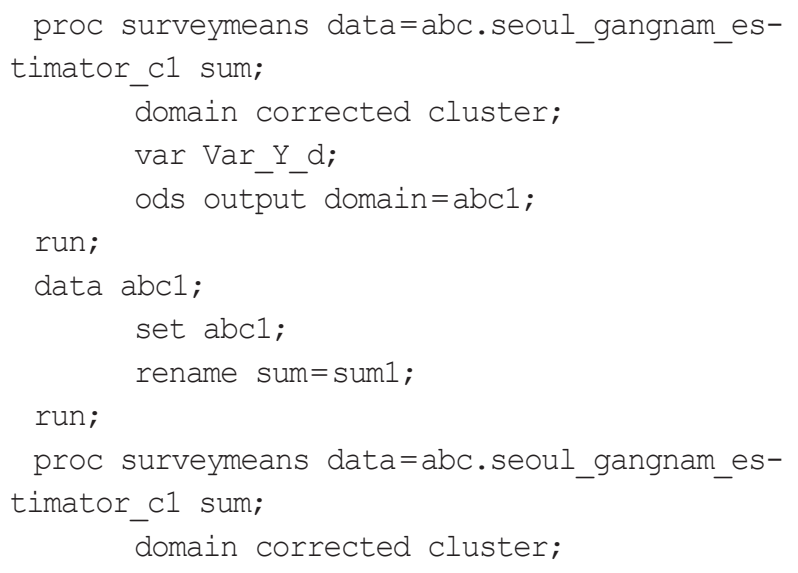


Table 7. Direct estimator and synthetic estimator calculation results for current smoking rates by dong

\begin{tabular}{|c|c|c|c|c|c|c|c|}
\hline Observation & Eup/myeon/dong & $\mathrm{n}$ & Y_d & Var_Y_d & Corrected cluster & Y_S & var_Y_s \\
\hline 1 & Gaepo 1-dong & 51 & 0.149 & 0.003 & 3 & 0.185 & 0.000485 \\
\hline 2 & Gaepo 2-dong & 52 & 0.163 & 0.004 & 3 & 0.188 & 0.000506 \\
\hline 3 & Gaepo 4-dong & 24 & 0.155 & 0.006 & 3 & 0.190 & 0.000511 \\
\hline 4 & Nonhyeon 1-dong & 41 & 0.383 & 0.007 & 1 & 0.265 & 0.000828 \\
\hline 5 & Nonhyeon 2-dong & 28 & 0.444 & 0.010 & 1 & 0.258 & 0.000742 \\
\hline 6 & Daechi 1-dong & 30 & 0.186 & 0.006 & 2 & 0.211 & 0.000528 \\
\hline 7 & Daechi 2-dong & 53 & 0.070 & 0.001 & 2 & 0.214 & 0.000541 \\
\hline 8 & Daechi 4-dong & 31 & 0.357 & 0.008 & 2 & 0.213 & 0.000595 \\
\hline 9 & Dogok 1-dong & 44 & 0.185 & 0.003 & 2 & 0.210 & 0.000548 \\
\hline 10 & Dogok 2-dong & 54 & 0.075 & 0.001 & 2 & 0.205 & 0.000506 \\
\hline 11 & Samsung 1-dong & 35 & 0.212 & 0.005 & 1 & 0.260 & 0.000694 \\
\hline 12 & Samsung 2-dong & 51 & 0.241 & 0.004 & 1 & 0.261 & 0.000737 \\
\hline 13 & Segok-dong & 25 & 0.108 & 0.003 & 3 & 0.190 & 0.000522 \\
\hline 14 & Sooseo-dong & 40 & 0.259 & 0.005 & 3 & 0.187 & 0.000516 \\
\hline 15 & Shinsa-dong & 36 & 0.218 & 0.005 & 1 & 0.251 & 0.000682 \\
\hline 16 & Apgujeong-dong & 36 & 0.156 & 0.005 & 1 & 0.245 & 0.000639 \\
\hline 17 & Yeoksam 1-dong & 58 & 0.365 & 0.004 & 2 & 0.219 & 0.000717 \\
\hline 18 & Yeoksam 2-dong & 55 & 0.267 & 0.005 & 2 & 0.211 & 0.000562 \\
\hline 19 & Ilwon 1-dong & 38 & 0.218 & 0.005 & 3 & 0.190 & 0.000513 \\
\hline 20 & Ilwon 2-dong & 44 & 0.167 & 0.003 & 3 & 0.188 & 0.000500 \\
\hline 21 & Ilwonbon-dong & 46 & 0.242 & 0.004 & 3 & 0.184 & 0.000490 \\
\hline 22 & Cheongdam-dong & 49 & 0.135 & 0.002 & 1 & 0.256 & 0.000692 \\
\hline
\end{tabular}

Y_d, direct estimate; Var_Y_d, variance of Y_d; Y_s, synthetic estimate; var_Y_s, variance of $Y_{-} s$.

var sumvar_Ys_Yd;

ods output domain=abc2;

run;

data abc3;

merge abc1 abc2;

by corrected cluster;

alpha2=1-( sum1/sum) ;

keep corrected cluster alpha2;

run;

proc sort data=abc.seoul_gangnam_estimator_ c1; by corrected cluster; run;

\section{(3) Calculation of composite estimates using the second weight} (Y_c2)

/*Composite estimator_2*/

data abc.seoul_gangnam_estimator_c2;

merge abc.seoul_gangnam_estimator_c1 abc3;

by corrected cluster;

Y_c2 $=($ alpha2*Y_d $\left.)+((1-a) p h a 2) * Y \_s\right)$;

Var_Y_c2 $=(($ alpha2*alpha2)*Var_y_

d) $+(((1-a l p h a 2) *(1-a l p h a 2))$ *Var_Y_s $)$;

run;

proc surveymeans data $=a b c$. seoul_gangnam_pop sum; domain eup/myeon/dong; var population; ods output domain=abc4; run;

proc surveymeans data=abc.seoul_gangnam_pop sum; domain corrected cluster; var population; ods output domain=abc5; run;

proc surveymeans data $=a b c$.seoul_gangnam_estimator_c2 sum; domain corrected cluster; var $\mathrm{N}$; ods output domain=abc6; run;

proc sort data=abc4; by eup/myeon/dong; run;

data abc5; set abc5; rename sum=cluster population; run;

proc sort data=abc5; by corrected cluster; run;

data abc6; set abc6; rename Sum=cluster sample size; run;

proc sort data=abc6; by corrected cluster;

run;

proc sort data=abc.seoul_gangnam_estimator_

c2; by eup/myeon/dong; run;

data abc.seoul_gangnam_estimator_c3_1; merge abc.seoul_gangnam_estimator_c2

abc4;

by eup/myeon/dong;

drop DomainLabel VarName VarLabel StdDev; rename sum=registered number of population;

run;

proc sort data=abc.seoul_gangnam_estimator_ 


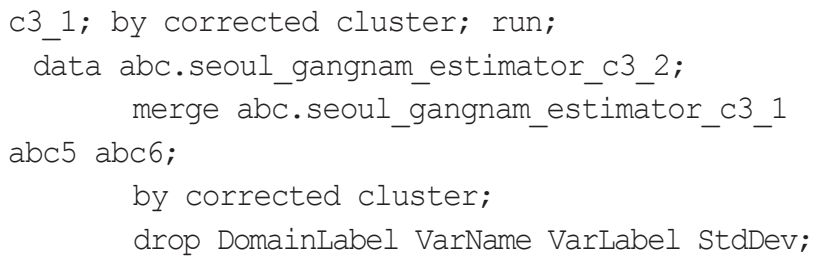

(4) Calculation of composite estimates using the third weight (Y_c3)

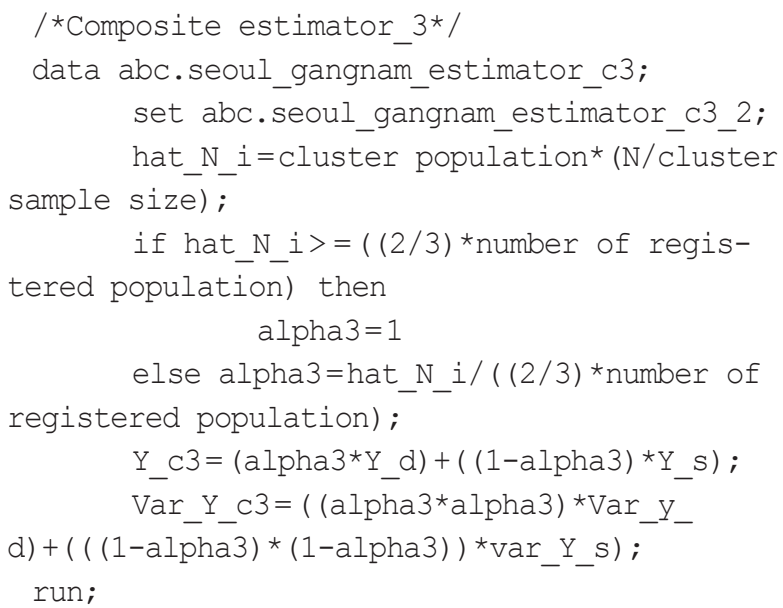

(5) Calculation of the composite estimates using the average weight of the first and third weights (Y_c4)

/*Composite estimator_4*/

data abc.seoul_gangnam_estimator_c4;

set abc.seoul_gangnam_estimator_c3;

alpha4 $=($ alpha1 + alpha3 $) / 2$

Y_c4 $=($ alpha4*Y_d $)+\left((1-a l p h a 4) * Y \_s\right) ;$

Var_Y_c4 $=(($ alpha4*alpha4)*Var_y_

d) $+(((1-a)$ pha 4$) *(1-a l p h a 4)) *$ var_Y_s $)$;

run;

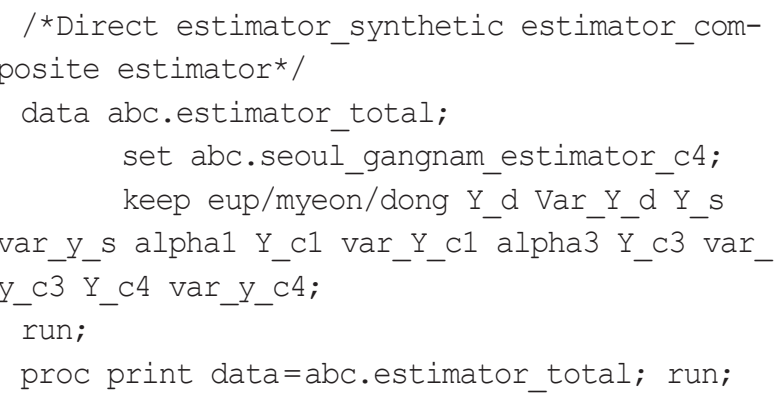

Table 8 outlines the results of calculating the current smoking rates of each of the 22 dongs applying the four types of composite estimates. While variances are found to be lower compared

Table 8. Results of estimating the current smoking rates by dong applying four types of composite estimate

\begin{tabular}{|c|c|c|c|c|c|c|c|c|c|c|c|}
\hline Eup/myeon/dong & $\mathrm{n}$ & Y_d & V_d & Y_c1 & V_c1 & Y_c2 & V_c2 & Y_c3 & V_c3 & $Y_{-} \mathrm{c} 4$ & V_c4 \\
\hline Nonhyeon 1-dong & 41 & 0.3832 & 0.0063 & 0.2787 & 0.0007 & 0.2787 & 0.0007 & 0.3832 & 0.0063 & 0.3310 & 0.0021 \\
\hline Nonhyeon 1-dong & 28 & 0.4439 & 0.0096 & 0.2718 & 0.0007 & 0.2802 & 0.0007 & 0.4439 & 0.0096 & 0.3579 & 0.0029 \\
\hline Samsung 1-dong & 35 & 0.2118 & 0.0052 & 0.2542 & 0.0006 & 0.2542 & 0.0006 & 0.2119 & 0.0052 & 0.2330 & 0.0018 \\
\hline Samsung 2-dong & 51 & 0.2407 & 0.0042 & 0.2584 & 0.0006 & 0.2591 & 0.0006 & 0.2407 & 0.0042 & 0.2495 & 0.0015 \\
\hline Shinsa-dong & 36 & 0.2180 & 0.0052 & 0.2476 & 0.0006 & 0.2475 & 0.0006 & 0.2180 & 0.0052 & 0.2328 & 0.0018 \\
\hline Apkujeong-dong & 36 & 0.1564 & 0.0051 & 0.2352 & 0.0006 & 0.2347 & 0.0006 & 0.1564 & 0.0051 & 0.1958 & 0.0017 \\
\hline Cheongdam-dong & 49 & 0.1351 & 0.0024 & 0.2288 & 0.0005 & 0.2421 & 0.0006 & 0.1351 & 0.0024 & 0.1820 & 0.0010 \\
\hline Daechi 1-dong & 30 & 0.1862 & 0.0059 & 0.2088 & 0.0005 & 0.2079 & 0.0005 & 0.1862 & 0.0059 & 0.1975 & 0.0018 \\
\hline Daechi 2-dong & 53 & 0.0703 & 0.0010 & 0.1642 & 0.0004 & 0.1965 & 0.0004 & 0.0703 & 0.0010 & 0.1173 & 0.0005 \\
\hline Daechi 4-dong & 31 & 0.3576 & 0.0077 & 0.2232 & 0.0006 & 0.2303 & 0.0006 & 0.3576 & 0.0077 & 0.2904 & 0.0023 \\
\hline Dogok 1-dong & 44 & 0.1855 & 0.0034 & 0.2064 & 0.0005 & 0.2068 & 0.0005 & 0.1855 & 0.0034 & 0.1960 & 0.0012 \\
\hline Dogok 2-dong & 54 & 0.0752 & 0.0011 & 0.1650 & 0.0003 & 0.1894 & 0.0004 & 0.0752 & 0.0011 & 0.1201 & 0.0005 \\
\hline Yeoksam 1-dong & 58 & 0.3655 & 0.0046 & 0.2390 & 0.0006 & 0.2369 & 0.0006 & 0.3655 & 0.0046 & 0.3023 & 0.0016 \\
\hline Yeoksam 2-dong & 55 & 0.2670 & 0.0053 & 0.2162 & 0.0005 & 0.2175 & 0.0005 & 0.2670 & 0.0053 & 0.2416 & 0.0017 \\
\hline Gaepo 1-dong & 51 & 0.1494 & 0.0030 & 0.1803 & 0.0004 & 0.1816 & 0.0004 & 0.1494 & 0.0030 & 0.1648 & 0.0011 \\
\hline Gaepo 2-dong & 52 & 0.1632 & 0.0041 & 0.1857 & 0.0005 & 0.1859 & 0.0005 & 0.1632 & 0.0041 & 0.1744 & 0.0014 \\
\hline Gaepo 4-dong & 24 & 0.1552 & 0.0069 & 0.1874 & 0.0005 & 0.1863 & 0.0005 & 0.1610 & 0.0048 & 0.1742 & 0.0016 \\
\hline Segok-dong & 25 & 0.1082 & 0.0036 & 0.1800 & 0.0005 & 0.1822 & 0.0005 & 0.1083 & 0.0036 & 0.1441 & 0.0012 \\
\hline Soosea-dong & 40 & 0.2596 & 0.0051 & 0.1933 & 0.0005 & 0.1940 & 0.0005 & 0.2596 & 0.0051 & 0.2264 & 0.0016 \\
\hline Ilwon 1-dong & 38 & 0.2184 & 0.0053 & 0.1930 & 0.0005 & 0.1934 & 0.0005 & 0.2184 & 0.0053 & 0.2057 & 0.0017 \\
\hline Ilwon 2-dong & 44 & 0.1670 & 0.0035 & 0.1860 & 0.0004 & 0.1865 & 0.0004 & 0.1670 & 0.0035 & 0.1765 & 0.0012 \\
\hline Ilwonbon-dong & 46 & 0.2416 & 0.0045 & 0.1894 & 0.0004 & 0.1895 & 0.0004 & 0.2416 & 0.0045 & 0.2155 & 0.0015 \\
\hline
\end{tabular}

Y_d, direct estimate; V_d, variance of $Y_{-} d$; $Y_{-} c 1$, first weight composite estimate; V_c1, variance of $Y_{-} c 1$; $Y \_c 2$, second weight composite estimate; $V_{-} c 2$, variance of $Y_{-} c 2 ; Y_{-} c 3$, third weight composite estimate; $V_{-} c 3$, variance of $Y_{-} c 3$; $Y_{-} c 4$, composite estimate using average weight of first and third weights; $V_{-} \mathrm{c} 4$, variance of $Y_{-} \mathrm{C} 4$. 
with direct estimates, the current smoking rate estimates vary widely depending on dong sample sizes, reflecting the estimatestabilizing feature of composite estimation. Viewed from the aspect of reducing the variance of direct estimates and correcting the bias of synthetic estimates, the fourth composite estimator is considered most effective in stabilizing the dong-level estimates.

\section{CONCLUSION}

This paper explained the procedure for producing health indicators at dong/eup/myeon-level (smaller than the designed domain) from the CHS data with the aim of producing health indicators at the level of health center district (designed domain), by applying small-area estimation to an example case of numerical estimation. The calculation procedures were presented using SAS codes, a method that is expected to be useful in cases in which statistics for smaller or detailed areas are to be produced from a survey conducted with the objective of producing statistics at a larger domain level.

\section{ACKNOWLEDGEMENTS}

This work was supported by the Research Program funded by the Korea Centers for Disease Control and Prevention (fund code 2014-P33001-00).

\section{CONFLICT OF INTEREST}

The authors have no conflicts of interest to declare for this study.

\section{SUPPLEMENTARY MATERIAL}

Supplementary material is available at http://www.e-epih.org/.

\section{REFERENCES}

1. Korea Centers for Disease Control and Prevention. Sample design for 2013 National Community Health Survey and monitoring sample survey. Cheongju: Korea Centers for Disease Control and Prevention; 2013, p. 9-11 (Korean).

2. Ghosh M, Rao JN. Small area estimation: an appraisal. Stat Sci 1994; 9:55-93.

3. Lee KO, Chung YS. Small-area estimation: research report. Daejeon: Statistics Korea; 2001, p. 70-76 (Korean).

4. Gonzalez Jr JF, Placek PJ, Scott C. Synthetic estimation in followback surveys at the National Center for Health Statistics. New York: Springer; 1996, p. 16-27.

5. Lee KO. Application of small-area estimation for estimating the number of the unemployed by si/gun/gu unit. Korean J Appl Stat 2000; 13:275-285 (Korean).

6. Korea Centers for Disease Control and Prevention. The study program (algorithm) to compute dong/eub/meon's statistics in Community Health Survey. Cheongju: Korea Centers for Disease Control and Prevention; 2014, p. 24-43 (Korean).

7. Korea Centers for Disease Control and Prevention. Community Health Survey raw data use guidelines. Cheongju: Korea Centers for Disease Control and Prevention; 2012, p. 28-35 (Korean).

8. Singh MP, Gambino J, Mantel HJ. Issues and strategies for small-area data. Surv Methodol 1994;20:3-22. 